\title{
Metabolic and Texture Features of PET/CT Scan to Predict Response in Patients with Locally Advanced Rectal Cancer Treated by Concurrent Neoadjuvant Chemoradiotherapy
}

mhsen aarabi

Alborz University of Medical Sciences

sajad pashutan shayesteh ( $\sim$ shayeste_sajad@yahoo.com )

Alborz University of Medical Sciences https://orcid.org/0000-0003-4122-0053

Afsaneh Alikhassi

Tehran University of Medical Sciences

Armaghan Fard Esfahani

Tehran University of Medical Sciences

Parham Geramifar

Tehran University of Medical Sciences

Ahmad Bitarafan-Rajabi

Iran University of Medical Sciences

\section{Research Article}

Keywords: PET/CT, Rectal cancer, Radiomics, Metabolic parameters

Posted Date: January 3rd, 2022

DOI: https://doi.org/10.21203/rs.3.rs-1203807/v1

License: (c) (i) This work is licensed under a Creative Commons Attribution 4.0 International License.

Read Full License 


\section{Abstract}

Background: Neoadjuvant chemoradiotherapy (nCRT) before surgical resection is the standard treatment for patients with locally advanced rectal cancer (LARC). Radiomics can be used as noninvasive biomarker for response prediction. The purpose of our study was to evaluate the potential of PET/CT texture features to predict the responses of LARC subjects treated with nCRT.

Methods: One week prior to nCRT, patients underwent Positron Emission Tomography/Computed Tomography (PET/CT) scan and then received concurrent nCRT. For each patient, intensity, shape and texture-based features were derived from PET/CT images using the IBEX software. The logistic regression classifier was used to identify the responders from non-responders.

Results: In this study, 23 patients with LARC were included. The patients' responses included 5 patients with Grade 0, 7 with Grade 1, 6 with Grade 2, and 5 with Grade 3 according to American Joint Committee on Cancer/College of American Pathologists (AJCC/CAP) pathologic grading. In quantitative texture features analysis, the dissimilarity feature had the highest performance [Area under Curve $(A U C)=0.65$ ] and in metabolic parameters analysis the best performance was for total lesion glycolysis (TLG; AUC= $0.61)$

Conclusions: In conclusion, performance of quantitative texture features is better than metabolic parameters but their performance should be improved.

\section{Introduction}

Colorectal cancer (CRC) is the third most common cancer worldwide and the second leading cause of cancer deaths in 2016 in the USA. Its risk is slightly lower in women than in men. One- third of the incidence is accounted for rectal cancer and approximately 39,220 new cases of rectal cancer with an about 5 -year overall survival rate of $65 \%$ will happen annually [1-5]. Nowadays, neoadjuvant chemoradiotherapy (nCRT) before surgical resection is the standard treatment for different tumor types and has been widely used for treating patients with locally advanced rectal cancer (LARC) [6, 7]. Determination of tumor response before treatment may significantly impact on designing an efficient strategy to treat cancer patients. Response to $\mathrm{nCRT}$ is considered as an important prognostic factor, but it significantly varies among patients. [8]. Prediction of the response to nCRT before treatment could be beneficial for LARC patients, but qualitative evaluation of response using medical images can be performed at the later stages of therapy. Because in qualitative evaluation, response to therapy is usually achieved by monitoring of the tumor length, area, and volume and these qualitative parameters are not apparent early during therapy $[9,10]$.

Radiomics is a new field of medical image study that aims to extract large number of high-throughput quantitative features that can be used as noninvasive, accurate and reliable biomarker for patients' response prediction and evaluation. Indeed, it focuses on the prediction of tumor behavior to treatment before therapy using quantitative analysis of tumor information $[11,12]$. But, when the number of derived 
features is more than samples number, the over-fitting is more likely to occur. Therefore, feature selection algorithms are used to identify robust and relevant features to improve accuracy of selected models. In this regard, previous studies have demonstrated how quantitative texture analysis and data mining methods may be used as predictive and prognostic biomarkers in lung adenocarcinoma, breast, head and neck squamous cell carcinoma, and prostate cancer [13-17]. Hence, the aim of our study was to investigate the potential of different radiomic features extracted from rectal PET/CT to find best predictive models in comparison to SUVmax, SUVmean, metabolic tumor volume (MTV), SUV $\geq 2.5$, and total lesion glycolysis (TLG).

\section{Methods}

\section{Patient characteristics}

This study was a prospective study of 23 patients (13 males and 10 females) who were treated at Radiation Oncology Department of Cancer Institute, Tehran University of Medical Sciences from October 2016 to April 2018. This study was approved by the Ethics Committee of Tehran University of Medical Sciences (Trial No. 95-04-227-33428, Ethical approval No. IR.TUMS.VCR.REC.1395.17.16) and Iran University of Medical Sciences (Ethics approval No. K17-137) and all patients signed an informed consent. Inclusion criteria were location of tumor about $15 \mathrm{~cm}$ above anal verge, tumor influence to perirectal fat (cT3-4) or lymph node involvement, age $\leq 80$ years, World health organization (WHO) performance situation of $0-2$, normal function of liver and renal system with normal complete blood count $(\mathrm{CBC})$ test, without any previous treatment for the disease. All patient received concurrent nCRT. They received 45-46 Gy external beam radiation in 23-25 fractions with $18 \mathrm{MV}$ photons to the tumor and locoregional disease including pre-sacral and internal iliac lymph nodes with a boost to the tumor for a total of 50-50.4 Gy. Also, concurrent capecitabine at $825 \mathrm{mg} / \mathrm{m} 2$ twice daily was used.

\section{Image acquisition}

Images were acquired using a Biograph 6 PET/CT scanner (Siemens Medical Solutions). Fields of view were $16 \mathrm{~cm}$ and $58 \mathrm{~cm}$ in axial and transverse directions, respectively. The CT scan system was a spiral 6 slice scanner with a $50 \mathrm{~cm}$ axial field of view. All patients were fasted at least 60 minutes before PET/CT scan with the blood glucose level less than $150 \mathrm{mg} / \mathrm{dL}$. About 60 minutes after the injection of 18F-FDG ( $5 \mathrm{MBq} / \mathrm{kg}$ per body weight), the patients were placed in the scanner and low-dose CT was performed from the base to the mid-thigh of the skull. The PET scan was acquired over the same body area. The CT data reconstructed with 256.256 matrix size and 5-mm slice thickness. The PET data were reconstructed with $128 \cdot 128$ matrix size and 5-mm slice thickness.

\section{Tumor segmentation}

For each primary cancer site, the three-dimensional gross tumor volume was drawn with two readers: a ten years' experience radiation oncologist, and a fifteen years' experience radiologist using a designated 
multi-platform, free and open-source software package for visualization and medical image computing (3D slicer, version 4.8.1; available at: http://slicer.org/).

\section{Preprocessing and texture feature extraction}

Before feature extraction and in order to noise reduction, intensity normalization and discretization, all PET/CT images were pre-processed by the method proposed by Collewet et al. and also discretization to $64 \mathrm{~Gy}$ level. In the Collewet et al. method, all image intensities are normalized between $\mu \pm 3 \sigma$, where $\mu$ is the mean value of gray-levels inside the region of interest (ROI), and $\sigma$ is the standard deviation $[18,19]$.

Also, to test the filter effect on radiomic model performance, we applied our feature extraction on PET/CT images with and without processing filters. The filters included LoG filter with sigma $0.5,1$, and 1.5. For feature extraction, we used the freely available radiomic software, imaging biomarker explorer (IBEX) that runs in Matlab platform.

Various radiomic features from different feature sets, including intensity, shape and texture-based features were extracted from processed and un-processed PET/CT images. Extracted features included shape features $(n=17)$, intensity histogram features $(n=9)$, intensity direct $(n=19)$, neighbor intensity difference $(n=5)$, co-occurrence matrix features $(C O M)(n=19)$, and gray level run-length matrix features (GLRLM; $n=11)[9,20]$.

\section{Response assessment}

For all patients, surgery was done 6-8 weeks after nCRT. After inking, the specimens were fixed in formalin for 24 hours. The whole tumor and mesorectum were serially sliced, axially, at $3 \mathrm{~mm}$ intervals, and treatment response was assessed according to the American Joint Committee on Cancer and College of American Pathologists (AJCC/CAP). The Dworak tumor regression grade (TRG) according to AJCC/CAP was established as follows: grade 0 [Pathologic Complete Response (PCR)], which is defined as no viable cancer cell; grade 1 (moderate response), representing single cells or small groups of cancer cells; grade 2 (minimal response), showing residual cancer outgrown by fibrosis; grade 3 (poor response), representing fibrosis outgrown by residual cancer [21-23].

\section{Univariate radiomic analysis}

For univariate analysis, significant radiomic features correlating with response were selected and a logistic regression model was used to find their predictive performance, which was based on Area Under Curve (AUC). Also, these features were compared between responder and non-responder groups. A paired $t$-test was performed to assess the significance of the differences between two groups. Statistical significance was assumed if a two-sided $P$ value $<0.05$. All of analysis were done using MedCalc statistical software.

\section{Result}

\section{Patients and response}


In the study, 23 patients (13 men; mean age of 60.01 years; age range of 31-80 years; 10 women; mean age of 52.2 years; age range of 27-67 years) with LARC were included. All patients underwent concurrent nCRT, followed by surgery. Patients' nCRT responses included 5 patients with grade 0,7 cases with grade 1,6 subjects with grade 2 , and 5 patients with grade 3 according to AJCC/CAP pathologic grading. Patient data and their response grade are shown in Table 1.

\section{Texture analysis}

Radiomic features with high correlation to therapy response were selected for PET/CT images separately. Our univariate analysis showed 11 radiomic features having high correlation with nCRT response by $\mathrm{PET} / \mathrm{CT}$ image. We found that all 6 top radiomic features were from co-occurrence matrix (COM) feature set. The results on AUC logistic regression classifier for these features are shown in Figure 1. The Dissimilarity feature had the highest performance $(A U C=0.68)$ followed by Sum Average $(A U C=0.66)$, Inter Quartile Range (AUC= 0.65), Cluster Tendency (AUC= 0.64), Variance (AUC=0.63), and Cluster Prominence $(A \cup C=0.61)$.

Results on selected radiomic feature values between responder and non-responder groups indicated that there was no significant difference between two groups in all features $(P>0.05)$.

Response prediction using metabolic features of PET/CT images indicated that the best result was for TLG (AUC= 0.61) followed by SUVmax (AUC= 59), SUVmean $(A \cup C=55)$, and MTV $(A U C=59)$.

\section{Discussion}

In the field of oncology, medical imaging has been playing a fundamental role, providing valuable data for a better management of cancer treatment and that PET/CT can produce three-dimensional images noninvasively, without the risks of ionizing radiation and with greater spatial and contrast resolution [24].

Radiomics extracts and mines large number of quantitative and distinct medical imaging features, and assumes that these quantitative medical imaging features are related to clinical information regarding tumor phenotype and may have clinical significance across different diseases. Thus, it provides valuable data for personalized therapy of tumors $[12,25]$.

The results of our study demonstrate that quantitative features from computerized texture analysis of LARC patients at the pretreatment PET/CT imaging were associated with response to nCRT and can be used as noninvasive biomarker for response prediction. $\mathrm{ML}$ methods can be reliable and accurate predictors and preprocessing LoG filter can improve their performance [26, 27].

Previous studies have shown the feasibility of radiomic modelling in LARC. Nakajo et al. used PET/CT images to examine whether the heterogeneity in primary tumor $18 \mathrm{~F}-\mathrm{FDG}$ can predict tumor response and prognosis of patients with esophageal cancer treated by chemoradiotherapy (CRT). The enrolled 52 patients with esophageal cancer underwent 18F-FDG-PET/CT studies before CRT. SUVmax, SUVmean, MTV, SUV $\geq 2.5, T L G$, and six heterogeneity parameters assessed by texture analysis were obtained. 
Patients were classified as responders or non-responders according to Response Evaluation Criteria in Solid Tumors. Texture features IV and SZV, and volumetric parameters MTV and TLG can predict tumor response, but all of them have limited value in prediction of prognosis of patients with esophageal cancer treated by CRT [28]. Also, Groheux et al. investigated the value of some clinicopathological parameters and 18FDG-PET/CT texture features to predict event-free survival (EFS) in the estrogen receptorpositive/human epidermal growth factor receptor 2-negative (ER+/HER2-) locally advanced breast cancer patients. PET/CT texture features and clinicopathological parameters were investigated before neoadjuvant chemotherapy. After completion of chemotherapy, all patients had breast surgery with axillary lymph node dissection, followed by radiation therapy and endocrine therapy for 5 years. Finally, the baseline PET parameters measured before neoadjuvant treatment were shown to have prognostic values in ER+/HER2- locally advanced breast cancer patients. After multivariate analysis, metabolically active tumor volume remained significant, while textural analysis of PET images did not have added value [29].

\section{Conclusion}

In conclusion, performance of quantitative texture features seems to be better than metabolic parameters but their performance should be improved. Texture features can be used as noninvasive biomarker for response prediction in LARC patient.

\section{Declarations}

\section{Disclosure of conflict of interest}

The authors of this manuscript declare no relationships with any companies, whose products or services may be related to the subject matter of the article.

\section{Funding:}

This study has received funding by the Alborz University of Medical Sciences, Karaj, Iran

\section{References}

1. Birkman EM, et al., Protein phosphatase 2A (PP2A) inhibitor CIP2A indicates resistance to radiotherapy in rectal cancer. Cancer Med, 2018.

2. Cossiolo DC, et al. POLYMORPHISM OF THE COX-2 GENE AND SUSCEPTIBILITY TO COLON AND RECTAL CANCER. Arq Bras Cir Dig. 2017;30(2):114-7.

3. Hathout L, Williams TM, Jabbour SK. The Impact of Novel Radiation Treatment Techniques on Toxicity and Clinical Outcomes In Rectal Cancer. Curr Colorectal Cancer Rep. 2017;13(1):61-72.

4. Siegel RL, Miller KD, Jemal A, Cancer statistics, 2016. CA Cancer J Clin, 2016. 66(1): p. 7-30. 
5. Martinez-Useros $\mathrm{J}$, et al. The potential predictive value of DEK expression for neoadjuvant chemoradiotherapy response in locally advanced rectal cancer. BMC Cancer. 2018;18(1):144.

6. Zhang $\mathrm{C}$, et al. Morphologic predictors of pathological complete response to neoadjuvant chemoradiotherapy in locally advanced rectal cancer. Oncotarget. 2018;9(4):4862-74.

7. Sathyakumar K, et al. Best MRI predictors of complete response to neoadjuvant chemoradiation in locally advanced rectal cancer. Br J Radiol. 2016;89(1060):20150328.

8. Noh GT, Kim NK. Genomic predictor of complete response after chemoradiotherapy in rectal cancer. Ann Transl Med. 2016;4(24):493.

9. Chee CG, et al. CT texture analysis in patients with locally advanced rectal cancer treated with neoadjuvant chemoradiotherapy: A potential imaging biomarker for treatment response and prognosis. PLoS One. 2017;12(8):e0182883.

10. Sun Y-S, et al. Locally advanced rectal carcinoma treated with preoperative chemotherapy and radiation therapy: preliminary analysis of diffusion-weighted MR imaging for early detection of tumor histopathologic downstaging. Radiology. 2009;254(1):170-8.

11. Chicklore S, et al. Quantifying tumour heterogeneity in 18F-FDG PET/CT imaging by texture analysis. Eur J Nucl Med Mol Imaging. 2013;40(1):133-40.

12. Aerts $\mathrm{HJ}$, et al. Decoding tumour phenotype by noninvasive imaging using a quantitative radiomics approach. Nature communications. 2014;5:4006.

13. Sacconi B, et al. Analysis of CT features and quantitative texture analysis in patients with lung adenocarcinoma: a correlation with EGFR mutations and survival rates. Clinical radiology. 2017;72(6):443-50.

14. Nie K, et al. Quantitative analysis of lesion morphology and texture features for diagnostic prediction in breast MRI. Acad Radiol. 2008;15(12):1513-25.

15. Ailianou A, et al. MRI with DWI for the Detection of Posttreatment Head and Neck Squamous Cell Carcinoma: Why Morphologic MRI Criteria Matter. American Journal of Neuroradiology; 2018.

16. Schieda N, et al. MRI assessment of pathological stage and surgical margins in anterior prostate cancer (APC) using subjective and quantitative analysis. J Magn Reson Imaging. 2017;45(5):1296303.

17. Saeys $Y$, Inza I, Larrañaga P. A review of feature selection techniques in bioinformatics Bioinformatics. 2007;23(19):2507-17.

18. Molina D, et al. Influence of gray level and space discretization on brain tumor heterogeneity measures obtained from magnetic resonance images. Comput Biol Med. 2016;78:49-57.

19. Vallières $M$, et al. A radiomics model from joint FDG-PET and MRI texture features for the prediction of lung metastases in soft-tissue sarcomas of the extremities. Phys Med Biol. 2015;60(14):5471.

20. $\mathrm{Ng} \mathrm{F}$, et al. Assessment of primary colorectal cancer heterogeneity by using whole-tumor texture analysis: contrast-enhanced CT texture as a biomarker of 5-year survival. Radiology. 2013;266(1):177-84. 
21. Haddad P, et al. Addition of oxaliplatin to neoadjuvant radiochemotherapy in MRI-defined T3, T4 or $\mathrm{N}+$ rectal cancer: a randomized clinical trial. Asia-Pacific Journal of Clinical Oncology. 2017;13(6):416-22.

22. Edge SB, Compton CC, The American Joint Committee on Cancer: the 7th edition of the AJCC cancer staging manual and the future of TNM. Annals of surgical oncology, 2010. 17(6): p. 1471-1474.

23. Nougaret $S$, et al. Neoadjuvant chemotherapy evaluation by MRI volumetry in rectal cancer followed by chemoradiation and total mesorectal excision: Initial experience. J Magn Reson Imaging. 2013;38(3):726-32.

24. Moffat BA, et al. Functional diffusion map: a noninvasive MRI biomarker for early stratification of clinical brain tumor response. Proc Natl Acad Sci U S A. 2005;102(15):5524-9.

25. Parmar C, et al. Machine learning methods for quantitative radiomic biomarkers. Scientific reports. 2015;5:13087.

26. Davnall F, et al. Assessment of tumor heterogeneity: an emerging imaging tool for clinical practice? Insights Imaging. 2012;3(6):573-89.

27. De Cecco $\mathrm{CN}$, et al. Texture analysis as imaging biomarker of tumoral response to neoadjuvant chemoradiotherapy in rectal cancer patients studied with 3-T magnetic resonance. Invest Radiol. 2015;50(4):239-45.

28. Nakajo $\mathrm{M}$, et al. Texture analysis of 18 F-FDG PET/CT to predict tumour response and prognosis of patients with esophageal cancer treated by chemoradiotherapy. Eur J Nucl Med Mol Imaging. 2017;44(2):206-14.

29. Groheux D, et al. 18 FDG-PET/CT for predicting the outcome in ER+/HER2-breast cancer patients: comparison of clinicopathological parameters and PET image-derived indices including tumor texture analysis. Breast Cancer Res. 2017;19(1):3.

\section{Figures}




\section{selected features AUC}

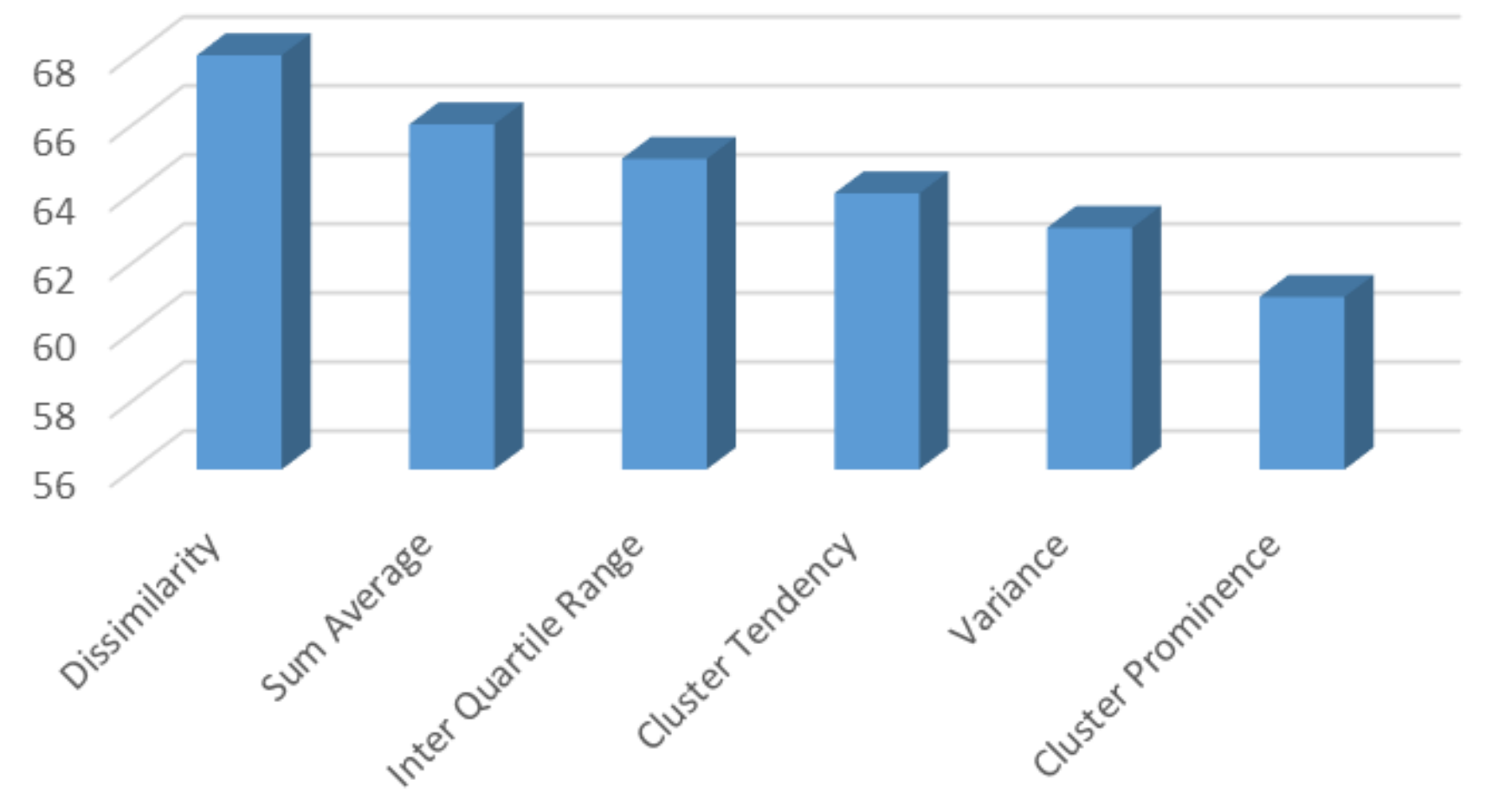

Figure 1

AUC of selected features in the univariate analysis for response prediction in LARC patients. 